Article

\title{
Determining the Jet Poloidal B Field and Black-Hole Rotation Directions in AGNs
}

\author{
Denise Gabuzda \\ Physics Department, University College Cork, Cork, Ireland; d.gabuzda@ucc.ie
}

Received: 21 October 2017; Accepted: 9 January 2018; Published: 12 January 2018

\begin{abstract}
It is theoretically expected that active galactic nucleus (AGN) jets should carry helical magnetic (B) fields, which arise due to the rotation of the central black hole and accretion disk combined with the jet outflow. The direction of the toroidal component of the helical $\mathbf{B}$ field $\mathbf{B}_{\varphi}$ is determined by the direction of the poloidal component $\mathbf{B}_{p}$ of the initial seed field that is "wound up" and the direction of rotation of the central black hole and accretion disk. The presence of the jet's helical B field can be manifest both through the presence of Faraday rotation gradients across the jet, and the presence of appreciable circular polarization, which comes about when linearly polarized emission from the far side of the jet is partially converted to circularly polarized emission as it passes through the magnetized plasma at the front side of the jet on its way towards the observer. When both of these properties are manifest, they can be used jointly with the jet linear polarization structure to uniquely determine both the direction of $\mathbf{B}_{p}$ and the direction of the central rotation. This technique has been applied to 12 AGNs. The results indicate statistically equal numbers of outward and inward $\mathbf{B}_{p}$ and of clockwise (CW) and counter-clockwise (CCW) rotations of the central black holes on the sky. However, they suggest that the directions of $\mathbf{B}_{p}$ and of the central rotation are coupled: CW/CCW central rotation is preferentially associated with inward/outward poloidal $\mathbf{B}$ field. This leads to a preferred orientation for the toroidal B-field component corresponding to inward current along the jet.
\end{abstract}

Keywords: active galactic nuclei; relativistic jets; magnetic fields; radio interferometry

\section{Introduction}

The centers of active galactic nuclei (AGNs) emit orders of magnitude more energy than is typical for a normal galaxy; this luminosity is too high to be explained by thermal processes such as star formation. The source of this energy is believed to be the conversion of gravitational energy to radiative and kinetic energy when matter is accreted onto a supermassive black hole at the galactic centre. While it is likely that all galaxies harbour black holes in the nuclei, the typical masses for such "normal" supermassive black holes may be of order $10^{6}$ solar masses, whereas the central black holes in AGNs may be $\sim 10^{9}$ solar masses. A sizeable minority of AGNs eject jets of relativistic plasma from their nuclei, which are often prominent at radio wavelengths through the synchrotron radiation they emit. This synchrotron radiation implies both the presence of appreciable magnetic (B) fields and mechanisms capable of accelerating charged particles (mainly electrons) to relativistic energies.

The synchrotron radiation observed for an ensemble of relativistic electrons moving in a $\mathbf{B}$ field will be linearly polarized if the $\mathbf{B}$ field is at least partially ordered. Linear-polarization observations provide direct information about the degree of order and orientation of the synchrotron $\mathbf{B}$ field. Synchrotron radiation can be polarized up to a maximum of about $75 \%$ for a completely ordered $\mathbf{B}$ field and an isotropic distribution of the pitch angles of the relativistic electrons [1]. The observed polarization is perpendicular to the $\mathbf{B}$ field in optically thin regions. High-resolution multi-wavelength radio observations can provide information about the radio spectrum of individual regions in the 
jet structure and Faraday rotation arising at various locations between the emission region and the observer.

\section{Faraday Rotation Gradients and Helical/Toroidal B Fields}

Faraday rotation is a rotation of the plane of polarization of an electromagnetic wave that occurs when it passes through a magnetized plasma. An electromagnetic wave can be described as the sum of right circularly polarized (RCP) and left circularly polarized (LCP) components. Due to asymmetry in the interactions between the local free charges and the RCP and LCP components of the polarized wave, these two components have different speeds of propagation through the magnetized medium. This introduces a delay between the RCP and LCP components, manifested as a rotation in the plane of polarization. The amount of rotation depends on the strength of the ambient magnetic field $\mathbf{B}$, the number density of thermal electrons in the plasma $n_{e}$, the charge $e$ and mass $m$ of the electron, and the wavelength of the radiation $\lambda$ (e.g., [2]):

$$
\chi=\chi_{o}+R M \lambda^{2} \quad R M=\frac{e^{3}}{8 \pi^{2} \epsilon_{o} m_{e}^{2} c^{3}} \int n_{e} \mathbf{B} \cdot d \mathbf{l}
$$

where $\chi$ is the observed polarization angle, $\chi_{0}$ is the intrinsic emitted polarization angle, $\epsilon_{o}$ is the permittivity of free space, and the integral is carried out over the line of sight from the source to the observer. While the magnitude of the rotation measure (RM) depends on both $n_{e}$ and the line-of-sight component of the magnetic field, the sign of the Faraday rotation is determined purely by the line-of-sight magnetic field-toward $(+)$ or away from $(-)$ the observer. The action of Faraday rotation can be identified through the $\lambda^{2}$ dependence of the observed polarization angle $\chi$. The predominant sources of Faraday rotation for extragalactic sources are magnetized plasma in our own Galaxy and in the immediate vicinity of the source. The former is typically up to a few tens of $\mathrm{rad} / \mathrm{m}^{2}$, which gives rise to rotations of no more than $\simeq 10^{\circ}$ at wavelengths of $6 \mathrm{~cm}$ or less.

As was pointed out by Blandford [3], the systematically changing line-of-sight component of a helical B field should give rise to a Faraday-rotation gradient across the jet. In fact, a possible RM gradient across the jet of NGC 6251 was noted in the early Very Large Array image by Perley et al. [4] in 1984, who suggested even then that it could be a sign of a helical B field associated with the kiloparsec-scale jet. No further observational work was done in this area until the report of a transverse RM gradient across the VLBI jet of 3C273 in 2002 [5].

Much work has been done since then, both in identifying additional AGNs displaying transverse RM gradients, and thereby evidence for helical jet B fields (e.g., [6-10]), and in carrying out Monte Carlo simulations in order to understand how to more accurately estimate the uncertainties in VLBI images and probe limits to the potential reliable detection of RM gradients [11-13].

\section{Circular Polarization and Helical/Toroidal B Fields}

The circular polarization (CP) of the radio emission of AGNs is much weaker than their linear polarization (LP), but is still detectable in at least some cases. Techniques for deriving CP information from data obtained with the Very Long Baseline Array (VLBA) were developed in [14,15]. These techniques have been used to derive parsec-scale CP measurements for a sample of 133 sources [16]. In the roughly $15 \%$ of these AGNs in which CP is detected, the CP is nearly always coincident with the VLBI core, with typical degrees of $\mathrm{CP}$ being a few tenths of a per cent. Comparisons with integrated data obtained earlier indicated that the sign of the $\mathrm{CP}$ for a given source usually remains constant on time scales of decades, although the CP sign does vary with time and/or wavelength in some cases (e.g., [17-19]). The groundbreaking work of [16] has since been augmented by multi-wavelength studies [17], as well as ongoing integrated monitoring measurements [18].

Various possible mechanisms for the generation of CP in relativistic astrophysical jets have been discussed in the literature [20-24]. Although it is difficult to prove conclusively, it is generally believed that the most likely mechanism for the generation of the observed circular polarization is the Faraday 
conversion of LP to CP in a magnetized plasma [25]. Faraday conversion does not require extreme or exotic conditions to operate, and is more efficient than the generation of $\mathrm{CP}$ by the synchrotron mechanism when the two are compared under similar conditions. In order for Faraday conversion to operate, the observed electric (E) vector for the LP must not be purely parallel or perpendicular to the B field in the conversion region projected onto the sky (i.e., Stokes $U$ must be non-zero). This led to the suggestion that a helical jet B-field geometry might provide a natural way to generate CP in AGN jets via Faraday conversion: a small fraction of the LP from the far side of the jet relative to the observer is converted to $\mathrm{CP}$ as it passes through magnetized plasma in or near the front side of the jet $[21,24]$. The presence of both poloidal and toroidal components in the helical $\mathbf{B}$ field ensures that the linear polarization $\mathbf{E}$ vector emitted at the far side of the jet has components both parallel and perpendicular to the $\mathbf{B}$ field at the near side of the jet (i.e., Stokes $U$ will be non-zero).

Gabuzda et al. [26] considered the sign of the CP generated through this process in a helical B field, which depends on the angle between the $\mathbf{E}$ field for the polarized synchrotron radiation emitted at the back of the helical field (relative to the observer) and the foreground B field, which in turn depends on the pitch angle and helicity of the helical field. This helicity is related to the directions of the toroidal $\mathbf{B}_{\varphi}$ and poloidal $\mathbf{B}_{p}$ components of the field. These relations are illustrated in Figure 1. Gabuzda et al. [26] identified eight AGNs for which VLBA-scale CP, transverse RM gradients, and clear LP structure were observed. They used the LP structure to infer the pitch-angle regime of the helical field (relatively small, longitudinal jet B field or relatively large, orthogonal jet B field), and the direction of the transverse RM gradient to infer the expected CP sign in the case of $\mathbf{B}_{p}$ pointing outward (a North pole) and inward (a South pole). They then compared the sign of CP expected for these two cases with the observed CP sign to deduce whether $\mathbf{B}_{p}$ was oriented outward or inward. This was a novel way to try to reconstruct the full three-dimensional picture of the helical jet $\mathbf{B}$ fields, but implied rather strange results: all the poloidal $\mathbf{B}$ field components in the jets seemed to correspond to South magnetic poles, which seemed improbable.

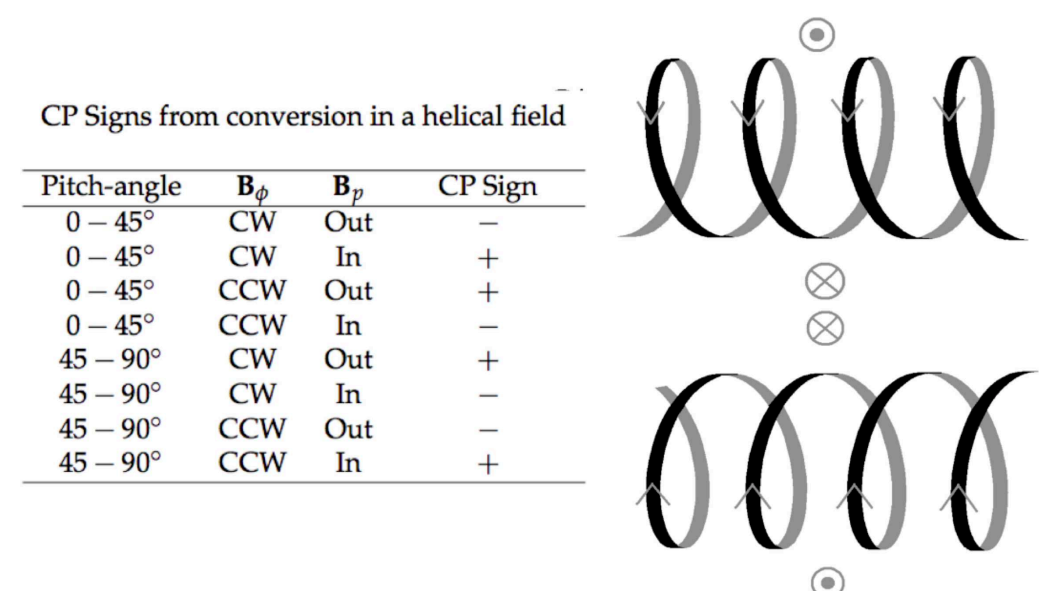

Figure 1. Left: table summarizing the circular polarization $(\mathrm{CP})$ signs produced by conversion in a helical $\mathbf{B}$ field. Right: Schematic showing the relationship between $\mathbf{B}_{\varphi}, \mathbf{B}_{p}$, and the helicity. $\mathbf{B}_{p}$ is pointing to the right in both cases, but the upper helix has right-handed helicity, giving rise to a counter-clockwise $(\mathrm{CCW}) \mathbf{B}_{\varphi}$, while the lower helix has left-handed helicity, giving rise to a clockwise $(\mathrm{CW}) \mathbf{B}_{\varphi}$.

\section{New Analysis}

A large number of new reports of reliable $(\geq 3 \sigma)$ transverse RM gradients have appeared in the literature over the past five years, whose significances have all been estimated using the error-estimation approach presented in [11]. This motivated us to re-examine the results of [26] and search for new sources that could be added to such an analysis. We have now determined that the 
analysis of [26] was unfortunately hampered by the lack at that time of a clear understanding of how to accurately estimate the reliability of transverse RM gradients detected by eye, as well as the weakness of the CP signals. Of the eight AGNs considered in [26], the transverse RM gradients in three sources and the $\mathrm{CP}$ detection in a fourth source were subsequently found to be not reliable (significant). On the other hand, an additional seven AGNs with $3 \sigma$ transverse RM gradients and reliable parsec-scale CP detections can now be identified. In addition, the RM gradient across the jet of $2230+114$ inferred in [26] based on a visual inspection of the RM map of [27] proved not to be significant, but a firm transverse RM gradient (in the opposite direction) was later reported in [11].

We present here the results of applying the technique of [26] to 12 AGNs (four from the original study and eight new cases). We have also extended this approach to use the inferred orientations of $\mathbf{B}_{\varphi}$ and $\mathbf{B}_{p}$ to deduce the direction of the rotation of the central black holes and their accretion disks. The procedure used was the same as that employed in [26], with the key difference that we had at our disposal reliable detections of statistically significant transverse RM gradients and CP in all 12 AGNs considered. As noted above, the sign of the CP generated via Faraday conversion in a helical $\mathbf{B}$ field is determined by the following parameters, which were derived as indicated:

- $\quad$ pitch-angle regime of the helical jet B field (large, $>45^{\circ}$, orthogonal jet $\mathbf{B}$ field; or small, $<45^{\circ}$, longitudinal jet $\mathbf{B}$ field)

- direction of $\mathbf{B}_{\varphi}$ (inferred from the direction of the transverse RM gradient)

- direction of $\mathbf{B}_{p}$ (unknown)

We used the sign of the CP observed on parsec scales at $15 \mathrm{GHz}[16,17]$ together with the observed linear polarization structure and the direction of the transverse RM gradient to infer the direction of $\mathbf{B}_{p}$. The direction of $\mathbf{B}_{\varphi}$ can be described as clockwise (CW) or counter-clockwise (CCW) on the sky. The directions of $\mathbf{B}_{\varphi}(\mathrm{CW}$ or $\mathrm{CCW})$ and of $\mathbf{B}_{p}$ (inward or outward) together indicate the direction of rotation of the central black hole and accretion disk on the sky (assuming $\mathbf{B}_{p}$ has been wound up by the rotation to yield $\mathbf{B}_{\varphi}$ ), which can also be described as CW or CCW on the sky. We assumed that we are viewing the jet at an angle that is less than $90^{\circ}$ in the rest frame of the jet, which will be true in most cases on statistical grounds. We have not explicitly taken into account any Faraday rotation between the back and front of the jet due to $\mathbf{B}_{p}$, but we would expect this to be small compared to the Faraday rotation produced by $\mathbf{B}_{\varphi}$, and to be approximately uniform across the jet.

\section{Results}

Our results are summarized in Table 1. Although the number of AGNs that can currently be included in this analysis is small, it is clear that our new analysis implies statistically equal numbers of outward (6) and inward (6) $\mathbf{B}_{p}$ and of CW (7) and CCW (5) rotations of the central black holes on the sky. This is consistent with our a priori expectations that there is no physical reason for a preference for a particular sense of rotation of the central regions of massive galaxies. In contrast, 9 of the 12 jets have inward currents; although this is not highly significant (it has about a $7 \%$ probability of arising by chance), it is nevertheless suggestive of an asymmetry.

Equivalently, the tentative predominance of inward currents provides evidence that the directions of $\mathbf{B}_{p}$ and of the central rotation are coupled, with $\mathrm{CW} / \mathrm{CCW}$ central rotation being preferentially associated with inward/outward $\mathbf{B}_{p}$. Such a correlation is actually predicted by the "cosmic battery" mechanism promoted in [28-30]. In this picture, Poynting-Robertson drag generates currents in the accretion disk in the direction of the rotation, coupling the directions of rotation and $\mathbf{B}_{p}$. When loops of B field generated by this mechanism are wound up by the differential rotation of the central black hole and accretion disk, they produce $\mathrm{CW} \mathbf{B}_{\varphi}$ (inward current) near the jet axis (dominates on small scales) and $C C W \mathbf{B}_{\varphi}$ (outward current) in a more extended region farther from the jet axis (dominates on large scales). Thus, the observed pattern is consistent with the significant operation of this "cosmic battery" in the accretion disks of AGNs, but the number of sources that could be included in this analysis is too small to enable firm conclusions based on these data alone. 
Table 1. Active galactic nuclei (AGN) jets with transverse rotation measure (RM) gradients and CP.

\begin{tabular}{lcccccccc}
\hline Source & $\begin{array}{c}\text { Pitch-Angle } \\
\text { Regime }\end{array}$ & $\mathbf{B}_{\boldsymbol{\varphi}}^{+}$ & Current $^{+}$ & $\begin{array}{c}\text { Observed } \\
\text { CP Sign }\end{array}$ & $\begin{array}{c}\text { Inferred } \\
\mathbf{B}_{p}\end{array}$ & $\begin{array}{c}\text { Inferred } \\
\text { Rotation }\end{array}$ & $\begin{array}{c}\text { RM } \\
\text { Reference }\end{array}$ & $\begin{array}{c}\text { CP } \\
\text { Reference * }\end{array}$ \\
\hline $0133+476$ & Large & CCW & Out & - & Out & CW & {$[10]$} & {$[16,17]$} \\
$0300+470$ & Large & CW & In & - & In & CW & {$[9]$} & {$[17]$} \\
$0333+321$ & Small & CW & In & - & Out & CCW & {$[7]$} & {$[16]$} \\
$0735+178$ & Large & CW & In & - & In & CW & {$[8]$} & {$[16]$} \\
$0945+408$ & Large & CW & In & + & Out & CCW & {$[9]$} & {$[16]$} \\
$1156+295$ & Large & CW & In & - & In & CW & {$[8]$} & {$[16,17]$} \\
$3 C 273$ & Small & CCW & Out & - & In & CCW & {$[11]$} & {$[16]$} \\
$1334-127$ & Small & CCW & Out & + & Out & CW & {$[8]$} & {$[16,17]$} \\
$1504-166$ & Large & CW & In & + & Out & CCW & {$[10]$} & {$[16]$} \\
$1633+382$ & Large & CW & In & - & In & CW & {$[7]$} & {$[16,17]$} \\
$1749+096$ & Large & CW & In & - & In & CW & {$[8]$} & {$[17]$} \\
$2230+114$ & Small & CW & In & - & Out & CCW & {$[11]$} & {$[17]$} \\
\hline
\end{tabular}

${ }^{+} \mathbf{B}_{\varphi}$ is inferred from the direction of the observed RM gradient. The current is inferred from $\mathbf{B}_{\varphi}$ using the right-hand rule. ${ }^{*}$ Numbers of references correspond to those in the bibliography.

\section{Conclusions}

We have carried out a joint analysis of the LP structures, transverse RM gradients, and CP signs of 12 AGNs, assuming that the observed CP is produced by Faraday conversion in the helical jet $\mathbf{B}$ fields that give rise to the transverse RM gradients. The fairly high fraction of AGNs with detectable parsec-scale CP that also have significant transverse RM gradients (12 of 44 AGNs, or 27\%) supports the hypothesis that the $\mathrm{CP}$ and the RM gradients have the same origin-a helical jet $\mathbf{B}$ field. This analysis has made it possible for the first time to determine the directions of both $\mathbf{B}_{\varphi}$ and $\mathbf{B}_{p}$, as well as the direction of the rotation of the central black hole and accretion disk.

Our results indicate statistically equal numbers of inward (6) and outward (6) poloidal B fields, and of central engines rotating CW (7) and CCW (5) on the sky, but hint at a predominance of inward currents on parsec scales $(9 / 12$ inferred currents are inward). This suggests that the directions of $\mathbf{B}_{p}$ and of the central rotation are correlated, with CW/CCW central rotation being preferentially associated with inward/outward $\mathbf{B}_{p}$. The existence of such a correlation, or coupling, is consistent with the action of the Poynting-Robertson battery described in [28-30].

The number of AGNs considered here is clearly too small a sample to demonstrate this correlation in any conclusive way; it is noteworthy, however, that Gabuzda et al. [10] have found a statistically significant excess of CW $\mathbf{B}_{\varphi}$ (inward current) inferred from parsec-scale transverse RM gradients across the jets of 47 AGNs, also very suggestive of the operation of this "cosmic battery" or some similar mechanism. An analysis like the one we have carried out here based on a larger sample of AGNs could help provide more conclusive evidence about the possible action of such a battery.

Conflicts of Interest: The authors declare no conflict of interest.

\section{References}

1. Pacholczyk, A.G. Radio Astrophysics; W. H. Freeman: San Franciso, CA, USA, 1970.

2. Burn, B.J. On the depolarization of discrete radio sources by Faraday dispersion. Mon. Not. R. Astron. Soc. 1966, 133, 67-83.

3. Blandford, R.D. Astrophysical Jets; Cambridge University Press: Cambridge, UK, 1993.

4. Perley, R.A.; Bridle, A.H.; Willis, A.G. High-resolution VLA observations of the radio jet in NGC 6251. Astrophys. J. Suppl. Ser. 1984, 54, 291-334.

5. Asada, K.; Inoue, M.; Uchida, Y.; Kameno, S.; Fujisawa, K.; Iguchi, S.; Mutoh, M. A Helical Magnetic Field in the Jet of 3C 273. Publ. Astron. Soc. Jpn. 2002, 54, L39-L43.

6. Gómez, J.L.; Marscher, A.P.; Jorstad, S.G.; Agudo, I.; Roca-Sogorb, M. On the Source of Faraday Rotation in the Jet of the Radio Galaxy 3C120. Astrophys. J. 2011, 733, 11-22. 
7. Gabuzda, D.C.; Reichstein, A.R.; O'Neill, E.L. Are spine-sheath polarization structures in the jets of Active Galactic Nuclei associated with helical magnetic fields? Mon. Not. R. Astron. Soc. 2014, 444, 172-184.

8. Gabuzda, D.C.; Knuettel, S.; Reardon, B. Transverse Faraday-rotation gradients across the jets of 15 Active Galactic Nuclei. Mon. Not. R. Astron. Soc. 2015, 450, 2441-2450.

9. Gabuzda, D.C.; Roche, N.; Kirwan, A.; Knuettel, S.; Nagle, M.; Houston, C. Parsec scale Faraday-rotation structure across the jets of nine active galactic nuclei. Mon. Not. R. Astron. Soc. 2017, 472, 1792-1801.

10. Gabuzda, D.C.; Nagle, M.; Roche, N. Are the Jets of Active Galactic Nuclei like Giant Coaxial Cables? Astron. Astrophys. 2018, submitted.

11. Hovatta, T.; Lister, M.L.; Aller, M.F.; Aller, H.D.; Homan, D.C.; Kovalev, Y.Y.; Pushkarev, A.B.; Savolainen, T. MOJAVE: Monitoring of Jets in Active Galactic Nuclei with VLBA Experiments. VIII. Faraday Rotation in Parsec-scale AGN Jets. Astrophys. J. 2012, 144, 105-138.

12. Mahmud, M.; Coughlan, C.P.; Murphy, E.; Gabuzda, D.C. Connecting magnetic towers with Faraday rotation gradients in active galactic nuclei jets. Mon. Not. R. Astron. Soc. 2013, 431, 695-709.

13. Murphy, E.; Gabuzda, D.C. The Innermost Regions of Relativistic Jets and Their Magnetic Fields. In Proceedings of the EPJ Web of Conferences, Granada, Spain, 10-14 June 2013.

14. Homan, D.C.; Wardle, J.F.C. Detection and Measurement of Parsec-Scale Circular Polarization in Four AGNS. Astrophys. J. 1999, 118, 1942-1962.

15. Homan, D.C.; Attridge, J.M.; Wardle, J.F.C. Parsec-Scale Circular Polarization Observations of 40 Blazars. Astrophys. J. 2001, 556, 113-120.

16. Homan, D.C.; Lister, M.L. MOJAVE: Monitoring of Jets in Active Galactic Nuclei with VLBA Experiments. II. First-Epoch 15 GHz Circular Polarization Results. Astrophys. J. 2006, 131, 1262-1279.

17. Vitrishchak, V.M.; Gabuzda, D.C.; Algaba, J.C.; Rastorgueva, E.A.; O’Sullivan, S.P.; O’Dowd, A. The 15-43 GHz parsec-scale circular polarization of 41 Active Galactic Nuclei. Mon. Not. R. Astron. Soc. 2008, 391, 124-135.

18. Angelakis, E. Full-Stokes, Multi-frequency Radio Polarimetry of Fermi Blazars: Monitoring and Modeling. Galaxies 2017, 5, 81 .

19. Aller, M.; Aller, H.; Hughes, P. The University of Michigan Centimeter-Band All Stokes Blazar Monitoring Program: Single-Dish Polarimetry as a Probe of Parsec-Scale Magnetic Fields. Galaxies 2017, 5, 75.

20. Macquart, J.-P.; Melrose, D.B. Scintillation-induced Circular Polarization in Pulsars and Quasars. Astrophys. J. 2000, 545, 798-806.

21. Laing, R.A.; Blundell, K.M. Particles and Fields in Radio Galaxies; Astronomical Society of the Pacific: San Francisco, CA, USA, 2002.

22. Wardle, J.F.C.; Homan, D.A. Theoretical Models for Producing Circularly Polarized Radiation in Extragalactic Radio Sources. Astrophys. Space Sci. 2003, 288, 143-153.

23. Beckert, T.; Falcke, H. Circular polarization of radio emission from relativistic jets. Astron. Astrophys. 2002, $388,1106-1119$.

24. Ensslin, T.A. Does circular polarisation reveal the rotation of quasar engines? Astron. Astrophys. 2003, 401, 499-504.

25. Jones, T.W.; O'Dell, S.L. Transfer of polarized radiation in self-absorbed synchrotron sources. I. Results for a homogeneous source. Astrophys. J. 1977, 214, 522-539.

26. Gabuzda, D.C.; Vitrishchak, V.M.; Mahmud, M.; O'Sullivan, S.P. Radio circular polarization produced in helical magnetic fields in eight active galactic nuclei. Mon. Not. R. Astron. Soc. 2008, 384, 1003-1014.

27. Taylor, G.B. Magnetic Fields in Quasar Cores. II. Astrophys. J. 2000, 533, 95-105.

28. Contopoulos, I.; Christodoulou, D.M.; Kazanas, D.; Gabuzda, D.C. The Invariant Twist of Magnetic Fields in the Relativistic Jets of Active Galactic Nuclei. Astrophys. J. Lett. 2009, 702, L148-L152.

29. Christodoulou, D.M.; Gabuzda, D.C.; Knuettel, S.; Contopoulos, I.; Kazanas, D.; Coughlan, C.P. Dominance of outflowing electric currents on decaparsec to kiloparsec scales in extragalactic jets. Astron. Astrophys. 2016, 591, A61-A71.

30. Contopoulos, I. Electric currents along astrophysical jets. Galaxies 2017, 5, 71.

(C) 2018 by the author. Licensee MDPI, Basel, Switzerland. This article is an open access article distributed under the terms and conditions of the Creative Commons Attribution (CC BY) license (http:/ / creativecommons.org/licenses/by/4.0/). 